\title{
Predictors of Failure Cardioversion for Recurrent Atrial Fibrillation Following Mitral Valve Surgery With Ablation
}

\author{
Hailong Cao, MD, PhD, ${ }^{1}$ Xin Chen, MD, ${ }^{2}$ Xiyu Zhu, MD,${ }^{1}$ Yining Yang, MD, ${ }^{2}$ Qing Zhou, MD, PhD, ${ }^{1}$ \\ Wei $\mathrm{Xu}, \mathrm{MD}, \mathrm{PhD},{ }^{2}$ Dongjin Wang, $\mathrm{MD}, \mathrm{PhD}^{1}$
}

\begin{abstract}
${ }^{1}$ Department of Thoracic and Cardiovascular Surgery, the Affiliated Drum Tower Hospital of Nanjing University Medical School, Nanjing, China; ${ }^{2}$ Department of Cardiology, the Affiliated Drum Tower Hospital of Nanjing University Medical School, Nanjing, China
\end{abstract}

\section{ABSTRACT}

Background: Electrical cardioversion (ECV) often is required for terminating recurrent atrial fibrillation $(\mathrm{AF})$ after surgical radiofrequency ablation in patients undergoing mitral valve surgery. However, ECV is unsuccessful in some cases. In this study, we aimed to identify possible predictors of failed ECV for recurrent atrial fibrillation following mitral valve surgery with concomitant radiofrequency ablation.

Methods: We enrolled 1,136 persistent AF patients with history of mitral valve surgery and concomitant radiofrequency ablation. Three-hundred-nineteen patients experienced recurrence of persistent AF and received ECV therapy. Comparison was made between patients with failed ECV (Failure group, $\mathrm{N}=68$ ) and successful ECV (Success group, $\mathrm{N}=251$ ).

Results: In multivariate regression analysis, age, pre-ECV loading-dose amiodarone, left atrial diameter, atrial flutter and time from surgery to ECV were independent predictors for outcomes of ECV. According to receiver operating characteristic curve analysis, the best threshold values of age, left atrial diameter and time from surgery to ECV for predicting failed ECV were 55.5 years, $64.5 \mathrm{~mm}$, and 90.5 days, respectively.

Conclusion: Older age, larger left atrium and longer time from surgery to ECV are independent predictors for failed $\mathrm{ECV}$ in this group. Compared with AF, atrial flutter is easier to be successfully terminated by ECV. Pre-ECV loadingdose amiodarone is helpful for successful ECV. These findings have important implications for identifying the kinds of patients to receive effective ECV.

\section{INTRODUCTION}

Persistent atrial fibrillation (AF) is present in $40 \%-50 \%$ of patients undergoing mitral valve surgery and has been

Received April 5, 2020; accepted April 14, 2020.

Correspondence: Professor Dongjin Wang, Department of Thoracic and Cardiovascular Surgery, the Affiliated Drum Tower Hospital of Nanjing University Medical School, 321 Zhongshan RD, Nanjing 210008, China; +862568182222-60722; fax: +862583105117 (e-mail: wangdongiin@njglyy. com or shuqu_1982@sina.com). identified as a risk factor for poor outcome after merely correcting underlying cardiac abnormality [Ruel 2005]. Maze $\mathrm{IV}$ procedure based on radiofrequency energy was confirmed effective in treating concomitant $\mathrm{AF}$ [Gillinov 2015] and was used worldwide. However, the failure rate within one year ranges from $17 \%$ to $26 \%$ after radiofrequency ablation [Gillinov 2015; Ad 2013]. For this kind of patient, we usually suggest antiarrhythmic drugs in clinic to try cardioversion or to control the ventricular rate to relieve symptoms.

According to a previous study, electrical cardioversion (ECV) for recurrent AF showed better results and stable recovery of sinus rhythm in patients undergoing concomitant surgical ablation, during mitral valve surgery [van Breugel 2014]. ECV is one of cornerstones for the rhythm control strategy of $\mathrm{AF}$, but it is associated with a considerable failure proportion up to $26 \%$ [Ecker 2018].

However, there is little evidence about the factors influencing the outcome of ECV in patients experiencing $\mathrm{AF}$ recurrence after mitral valve surgery and concomitant radiofrequency ablation. Herein, we aimed to reveal potential predictors of $\mathrm{ECV}$ failure for recurrent $\mathrm{AF}$ in this kind of patient.

\section{MATERIALS AND METHODS}

Patient enrollment: 1,136 persistent $\mathrm{AF}$ patients received concomitant radiofrequency ablation, during mitral valve surgery at the Affiliated Drum Tower Hospital of Nanjing University Medical School between April 2013 and May 2018. Four-hundred-fourteen cases experienced early recurrence of $\mathrm{AF}$ or atrial flutter (AFL), among which recurrence was terminated with loading-dose amiodarone in 95 cases. Threehundred-nineteen persistent $\mathrm{AF}$ or $\mathrm{AFL}$ cases received $\mathrm{ECV}$ with written informed consent. The study was conducted according to the Helsinki Declaration and approved by the ethics committee of Nanjing University.

Surgical technique: All operations were carried out on cardiopulmonary bypass with cardiac arrest. The mitral valve surgery was performed through a standard left atriotomy or an atrial septal incision. Different techniques were accomplished, according to the underlying mitral valve and other diseases. (Table 1) With regard to AF surgery, pulmonary vein isolation was carried out with a bipolar radiofrequency clamp (Medtronic, Minneapolis, Minn.). The left and right 
Table 1. Clinical characteristics of study population

\begin{tabular}{|c|c|c|c|}
\hline Variables & Failure & Success & $P$ \\
\hline \multicolumn{4}{|l|}{ Basic data } \\
\hline Patient number $(\mathrm{N})$ & 68 & 251 & - \\
\hline Gender, M/F (N) & $25 / 43$ & $112 / 139$ & .246 \\
\hline Age (years) & $59.6 \pm 8.5$ & $53.3 \pm 10.5$ & $<.001$ \\
\hline Body mass index $\left(\mathrm{kg} / \mathrm{m}^{2}\right)$ & $23.2 \pm 2.7$ & $22.9 \pm 2.7$ & .573 \\
\hline Hypertension (N) & 28 & 100 & .842 \\
\hline Diabetes $(N)$ & 13 & 48 & .999 \\
\hline \multicolumn{4}{|l|}{ Operative data } \\
\hline Mitral valve pathology $(\mathrm{N})$ & & 0.553 & \\
\hline Rheumatic & 46 & 152 & \\
\hline Degenerative & 18 & 79 & \\
\hline Ischemic or Functional & 4 & 20 & \\
\hline Mitral lesion $(\mathrm{N})$ & & 0.532 & \\
\hline Stenosis & 35 & 111 & \\
\hline Insufficiency & 22 & 98 & \\
\hline Mixed & 11 & 42 & \\
\hline \multicolumn{4}{|l|}{ Types of valvular surgery $(\mathrm{N})$} \\
\hline Mitral valve replacement/repair & $51 / 17$ & $172 / 79$ & .302 \\
\hline Aortic valve replacement or repair & 14 & 55 & .814 \\
\hline Tricuspid valve repair & 52 & 191 & .949 \\
\hline Combined other surgeries $(\mathrm{N})$ & & 0.833 & \\
\hline Coronary artery bypass grafting & 5 & 31 & \\
\hline Congenital heart diseases & 1 & 5 & \\
\hline Others & 0 & 2 & \\
\hline \multicolumn{4}{|l|}{ Pre-ECV data } \\
\hline Beta-blocker $(\mathrm{N})$ & 54 & 219 & .103 \\
\hline Amiodarone $(\mathrm{N})$ & 20 & 167 & $<.001$ \\
\hline \multicolumn{4}{|l|}{ Echocardiography before ECV } \\
\hline LVDd (mm) & $55.0 \pm 9.4$ & $53.8 \pm 10.5$ & .388 \\
\hline LVDs (mm) & $36.3 \pm 7.8$ & $36.1 \pm 8.2$ & .830 \\
\hline $\mathrm{EF}(\%)$ & $50.3 \pm 6.9$ & $52.7 \pm 5.9$ & .013 \\
\hline LAD (mm) & $58.1 \pm 10.8$ & $52.7 \pm 7.8$ & $<.001$ \\
\hline Ventricular rate (beats/min) & $83 \pm 13$ & $98 \pm 26$ & $<.001$ \\
\hline AF/Atrial flutter (N) & $63 / 5$ & $168 / 83$ & $<.001$ \\
\hline Time from surgery to ECV (dys) & $101 \pm 54$ & $71 \pm 25$ & $<.001$ \\
\hline
\end{tabular}

Values are presented as mean \pm SD or number of patients. ECV, electrical cardioversion; EF, ejection fraction; LAD, left atrial diameter; LVDd, left ventricular end-diastolic diameter; LVDs, left ventricular end-systolic diameter.

isthmus lesions always were performed with a radiofrequency pen (Medtronic, Minneapolis, Minn.). Moreover, the other left and right lesion lines were completed by a bipolar radiofrequency clamp. Finally, the left and right appendages and
Table 2. Multivariable predictors of failure of ECV for $\mathrm{AF}$ recurrence after surgical ablation

\begin{tabular}{lcccc}
\hline \multirow{2}{*}{ Variables } & \multicolumn{2}{c}{ Odds ratio } & $95 \% \mathrm{Cl}$ & $P$ \\
& Low limit & Upper limit & & \\
\hline Age & 1.069 & 1.032 & 1.108 & $<.001$ \\
Pre-ECV amiodarone & 0.217 & 0.104 & 0.450 & $<.001$ \\
Left atrial diameter & 1.052 & 1.005 & 1.101 & .031 \\
Atrial flutter & 0.227 & 0.077 & 0.674 & .008 \\
Time from surgery to ECV & 1.022 & 1.012 & 1.032 & $<.001$
\end{tabular}

Marshall ligament were excised in all patients. More details about surgical techniques previously have been reported [van Breugel 2014].

Postoperative management and follow up: After operation, unless contraindicated or indicating bradycardia, patients received amiodarone and/or beta-blocker to maintain sinus rhythm and control ventricular rate. According to the Heart Rhythm Society Consensus document on AF [Calkins 2007], the initial 90 days after the ablation were considered as the blanking period. Within the 3 months after discharge, all patients were required to visit clinic every week to complete a cardiac rhythm evaluation and coagulation test.

In the present study, $\mathrm{AF}$ recurrence was defined as atrial tachyarrhythmia (AF or AFL) lasting 30 seconds or longer. All patients with AF recurrence would receive drug cardioversion unless contraindicated. One week later, the patients with failed spontaneous or drug cardioversion with persistent $\mathrm{AF}$ or AFL ( $\geq 7$ days) were recommended to receive ECV.

After the blanking period, patients received monthly follow up for medication adjustment and cardiac rhythm evaluation with 12-lead surface electrocardiograms. Patients experiencing persistent $\mathrm{AF}$ recurrence were admitted to directly receive ECV.

Electrical cardioversion: A transesophageal echocardiography was performed to rule out intra-atrial thrombi before ECV in all patients. These patients were admitted in Department of Cardiology to carry out ECV with continuous electrocardiographic monitoring and full equipment for cardiopulmonary resuscitation. Blood pressure intermittently was measured during the procedure, while pulse oximetry and cardiac rhythm were continuously monitored throughout. Oxygen supplementation and ventilation support were given, if necessary. Twelve-lead electrocardiograms were recorded before and after the procedure to verify restoration of sinus rhythm. Pacing pads anteriorly were placed over the chest and posteriorly in the interscapular area. After given intravenous propofol $2.0 \mathrm{mg} / \mathrm{kg}$, an R-wave synchronized direct-current biphasic shock was delivered, with an initial energy of $200 \mathrm{~J}$ and then, if the first shock was ineffective, of $300 \mathrm{~J}$ up to 360 $\mathrm{J}$, according to the body weight and transthoracic impedance. After the procedure, patients were monitored by telemetry for at least 24 hours and discharged. Successful cardioversion was defined as maintenance of stable sinus rhythm for $>24$ hours after cardioversion. 
Table 3. Predictive values of failure of ECV for AF recurrence after surgical ablation by ROC curve

\begin{tabular}{|c|c|c|c|c|c|}
\hline Age & 55.5 yrs & $0.667(0.599 \sim 0.735)$ & 70.6 & 61.0 & 63.0 \\
\hline Time surgery to ECV & 90.5 dys & $0.677(0.596 \sim 0.757)$ & 50.0 & 82.9 & 75.9 \\
\hline
\end{tabular}

Best Cut-off value is equal to the biggest Youden index.

Youden index= Sensitivity+ Specificity-1. AUC, area under the curve

Statistical analysis: Statistical analysis was executed with SPSS 22.0 package (SPSS Inc., Chicago, Ill.). For the comparison between the 2 groups, Student's t-test (normally distributed) or Mann-Whitney test (non-normally distributed) was used for continuous variables, and c2 test was utilized for categorical variables. To reveal predictors of failure of ECV, multivariable binary logistic regression, including univariateanalyzed variables with a $P$ value $<.1$ was used. Therefore, outcome of ECV was defined as dependent variable. A $P$ value $<.05$ was defined as statistically significant.

Odds ratio (OR) and 95\% confidence interval (CI) were calculated. Youden index (Sensitivity+ Specificity-1) of receiver operating characteristic (ROC) curve was calculated to single out the best cutoff values of age, left atrial diameter (LAD) and time from surgery to ECV predicting failed ECV. Sensitivity, specificity and accuracy were investigated by the Fisher's exact test. Cox proportional hazards analysis was applied to calculate the effects of age, left atrial diameter and time from surgery to $\mathrm{ECV}$ at best cut-off value for predicting failure of ECV.

\section{RESULTS}

Patient characteristics: The 319 patients were divided into 2 groups, according to successful ECV or not (Failure group $\mathrm{N}=68$; Success group $\mathrm{N}=251$, see Table 1). The Failure group was older than the Success group. No significant differences were found between the 2 groups, in terms of sex distribution, body mass index, proportion of hypertension and diabetes, mitral valve pathology and lesion, and types of surgery. The Failure group had a less proportion of pre-ECV loading-dose amiodarone than the Success group, while usage of beta-blocker was comparable. The echocardiographic LAD was larger in Failure than Success, but ejection fraction was lower in Failure than Success. The systolic and diastolic size of the left ventricle did not differ between the 2 groups. Moreover, ventricular rate before ECV was lower in Failure than Success. The Failure group had a lower proportion of AFL than the Success group. The time from surgery to ECV was longer in Failure than Success.

Predictors for outcomes of ECV: Univariate analysis showed age, pre-ECV loading-dose amiodarone, ejection fraction, LAD, ventricular rate before ECV, atrial flutter and time from surgery to ECV were predictors for outcomes of ECV (Supplementary Table 1).
By multiple stepwise regression analysis, age, pre-ECV loading-dose amiodarone, LAD, atrial flutter and time from surgery to $\mathrm{ECV}$ were independent predictors for outcomes of ECV (Table 2).

Predictive values of failed ECV: According to the ROC curve analysis, the best threshold value of age for predicting failed ECV was 55.5 years old (Table 3). This cutoff value showed $70.6 \%$ sensitivity, $61.0 \%$ specificity, and $63.0 \%$ accuracy. The best threshold value of LAD for predicting failed ECV was $64.5 \mathrm{~mm}$ with $36.8 \%$ sensitivity, $93.6 \%$ specificity, and $81.5 \%$ accuracy. Furthermore, the best threshold value of time from surgery to ECV for predicting failed ECV was 90.5 days with $50.0 \%$ sensitivity, $82.9 \%$ specificity, and $75.9 \%$ accuracy.

In addition, according to Cox proportional hazards analysis at best cut-off value of these factors, age $\geq 55.5$ years old was showed 3.747 (2.098-6.692) times risk to experience failed ECV compared with age $<55.5$ years old. The ORs of $\mathrm{LAD} \geq 64.5 \mathrm{~mm}$ and time from surgery to $\mathrm{ECV} \geq 90.5$ days for predicting failed ECV were 8.539 (95\% CI: 4.212-17.312) and 4.837 (95\% CI: 2.714-8.620), compared with LAD $<64.5 \mathrm{~mm}$ and time from surgery to ECV $<90.5$ days, respectively (Table 4).

\section{DISCUSSION}

Surgical radiofrequency ablation of valvular AF is a wellaccepted strategy to treat concomitant AF. The possibility to restore sinus rhythm can increase the chance to perform valve repair and valve replacement with a biological prosthesis to avoid lifelong therapy with warfarin, particularly in old patients, when it is preferable to reduce the higher risk of bleeding. Therefore, survival, functional status and life quality will be much better in patients in sinus rhythm [Ad 2013]. We found that age, LAD, pre-ECV loading-dose amiodarone, AFL and time from surgery to $\mathrm{ECV}$ were independent predictors for outcomes of ECV.

Older age was expected and may reflect the severity of arrhythmic burden and atrial structural remodeling. This is why the incidence of AF gradually increases with age [Guo $2015]$. According to a recent study, advanced age $(\mathrm{OR}=1.03$; $95 \%$ CI $1.02-1.05, P<.001)$ was the only independent predictor of unsuccessful cardioversion [Grönberg 2015]. This was consistent with our results, which also indicated that age $\geq 55.5$ years old showed an average 3.747 times risk to 
Table 4. Predictors of failure of ECV for AF recurrence after surgical ablation at best cut-off values

\begin{tabular}{lcccc}
\hline \multirow{2}{*}{ Variables } & \multicolumn{2}{c}{ Odds ratio } & $95 \% \mathrm{Cl}$ & $P$ \\
& Low limit & Upper limit & & \\
\hline Age $\geq 55.5$ yrs & 3.747 & 2.098 & 6.692 & $<.001$ \\
LAD $\geq 64.5 \mathrm{~mm}$ & 8.539 & 4.212 & 17.312 & $<.001$ \\
Time to ECV $\geq 90.5$ dys & 4.837 & 2.714 & 8.620 & $<.001$
\end{tabular}

experience failed ECV compared with age $<55.5$ years old in our population. It might be attributed to more severe atrial electrical and structural remodeling and longer duration of $\mathrm{AF}$ for patients with advanced age.

The effect of left atrial size on the recurrence of $\mathrm{AF}$ previously has been confirmed [Ad 2014]. Marchese et al [Marchese 2011] and also Akdemir et al [Akdemir 2013] concluded that larger LAD before ECV was strongly associated with higher risk of AF recurrence. Van Breugel et al showed that $\mathrm{LAD} \geq 45.5 \mathrm{~mm}$ before ECV was an independent predictor for the AF recurrence after ECV [van Breugel 2014]. However, the population was small and most of them did not receive ablation procedure. In fact, atrial size markedly was more reduced in patients with associated ablation than in those with isolated mitral valve surgery as a result of a significantly higher reverse remodeling in the left atrium following surgical radiofrequency lesions [Ad 2014]. Atrial remodeling finally could result in many structural, electrical, metabolic, and neurohormonal consequences, which are mostly reversible [Marchese 2011; Casaclang-Verzosa 2008]. Determining an irreversible threshold for atrial remodeling in AF may be difficult, but achievable. Herein, we presented a cut-off value at $64.5 \mathrm{~mm}$ of pre-ECV echocardiographic LAD could be a used as a clinical value for distinguishing failed ECV based on the undermining microscopic irreversible changes. It might slightly expand the previous indications for the efficacy of ECV after ablation.

One previous study focusing on persistent AF has shown higher success rate of ECV during antiarrhythmic treatment [Crijns 2014]. Consistently, we observed a positive association of pre-ECV loading-dose amiodarone on success of ECV in our patient cohort. It is reasonable to assume that in the present patient cohort, the use of amiodarone reflects higher arrhythmic burden. Therefore, from our experience, the pre-treatment with oral amiodarone before ECV improves the reversion rate in patients with $\mathrm{AF}$ recurrence after mitral surgery. Mechanisms that may lead to the suppression of $\mathrm{AF}$ recurrence and reduced defibrillation threshold include the suppression of premature atrial contractions and atrial or pulmonary vein tachycardia, as well as a reduction of atrial refractoriness heterogeneity [Crijns 1991].

Consequently, the use of amiodarone should be, in our opinion, highly recommended in these patients before ECV.

AFL often is associated with rapid ventricular rates, which may cause severe symptoms. Therefore, cardioversion of the arrhythmia may be the best initial treatment and may be accomplished by pharmacologic or electrical means. Despite the well-known efficacy of catheter ablation in AFL treatment, ECV still plays an important role for its high reversion rate in patients who refuse catheter ablation or experience AFL recurrence after surgical ablation [van Breugel 2014]. As anticipated, the present study showed that the type of underlying arrhythmia is a very important determinant of outcome of ECV. This means that in patients presenting with AFL, ECV may be considered in almost all instances. This favorable arrhythmia prognosis is almost unaffected by other clinical parameters. In contrast, in $\mathrm{AF}$, arrhythmia outcome is highly influenced by many clinical factors [Van Gelder 1991].

According to our analysis, it emerges that the time from surgery to ECV also is a matter of utmost importance for predicting failed ECV. As well as we know, early AF recurrence after surgical ablation is explained by changes induced in atrial electrophysiology by myocardial edema and inflammatory response to surgery [Ishii 2004]. But the genesis of late recurrence might be attributed to lesion incompleteness [Maroto 2011]. Since early recurrence probably is inflammatory-mediated, we could expect that ECV performed after a 3-month blanking period must be associated with a lower success rate. In agreement with this speculation, we found that time from surgery to ECV significantly was longer in the Failure group than the Success group, and the best cut-off value for predicting failed ECV was 90.5 days. This finding strongly supports the hypothesis that cardioversion should be performed within the 3-month blanking period from surgery. It might render the ECV ineffective for irreversible anatomical and electrophysiological changes in the atrial conduction tissue after this period [Voskoboinik 2017].

Limitations: First, the nonrandomized design might affect the results because of unmeasured confounders, procedure bias or detection bias, despite the use of rigorous statistical adjustment. Second, since different combinations of antiarrhythmic drugs and various doses had been given to the postoperative patients and patients with $\mathrm{AF}$ recurrence according to their clinical states, it was difficult to analyze the influence of combinations, duration and doses to the rhythm. Third, the follow up was limited to a maximum of 284 days after surgical ablation and the demonstration of long-term maintenance rate of sinus rhythm and the outcomes of ancillary cardioversion would have been more desirable. Moreover, all procedures were performed in a single institution. A related potential bias may result from the reliance on self-reporting. So a multi-center study with a large population is warranted to confirm our findings.

\section{CONCLUSION}

Our data suggest that ECV of recurrent AF following mitral valve surgery and concomitant radiofrequency ablation is an effective procedure. Age $\geq 55.5$ years old, LAD $\geq 64.5 \mathrm{~mm}$ and time from surgery to ECV $\geq 90.5$ days indicate a worse outcome of ECV. AFL is easy to be successfully terminated by ECV. Pre-ECV loading-dose amiodarone is helpful for successful ECV. These findings may be helpful, when 
choosing treatment strategy in patients with $\mathrm{AF}$ recurrence after surgery.

\section{ACKNOWLEDGEMENT}

This work was supported in part by Fundamental Research Funds for the Central Universities [YG1903002], Jiangsu Provincial Medical Youth Talent [QNRC2016034], Jiangsu Province Health Department Program Grant [Z201411], Key Project supported by Medical Science and technology development Foundation, Nanjing Department of Health [YKK17066].

\section{REFERENCES}

Ad N, Henry L, Massimiano P, Pritchard G, Holmes SD. 2013. The state of surgical ablation for atrial fibrillation in patients with mitral valve disease. Curr Opin Cardiol 28:170-180.

Ad N, Holmes SD. 2014. Prediction of sinus rhythm in patients undergoing concomitant Cox maze procedure through a median sternotomy. $\mathrm{J}$ Thorac Cardiovasc Surg 148:881-886.

Ad N, Holmes SD, Massimiano PS, et al. 2013. The effect of the Coxmaze procedure for atrial fibrillation concomitant to mitral and tricuspid valve surgery. J Thorac Cardiovasc Surg 146:1426-1434.

Akdemir B, Altekin RE, Küçük M, et al. 2013. The significance of the left atrial volume index in cardioversion success and its relationship with recurrence in patients with non-valvular atrial fibrillation subjected to electrical cardioversion: a study on diagnostic accuracy. Anadolu Kardiyol Derg 13:18-25.

Calkins H, Brugada J, Packer DL, et al. 2007. HRS/EHRA/ECAS expert consensus statement on catheter and surgical ablation of atrial fibrillation: recommendations for personnel, policy, procedures and follow-up. A report of the Heart Rhythm Society (HRS) Task Force on catheter and surgical ablation of atrial fibrillation. Heart Rhythm 4:816-861.

Casaclang-Verzosa G, Gersh BJ, Tsang TS. 2008. Structural and functional remodeling of the left atrium: clinical and therapeutic implications for atrial fibrillation. J Am Coll Cardiol 51:1-11.

Crijns HJ, Van Gelder IC, Van Gilst WH, et al. 1991. Serial antiarrhythmic drug treatment to maintain sinus rhythm after electrical cardioversion for chronic atrial fibrillation or atrial flutter. Am J Cardiol 68:335-341.

Crijns HJ, Weijs B, Fairley AM, et al. 2014. Contemporary real life cardioversion of atrial fibrillation: Results from the multinational RHYTHM-AF study. Int J Cardiol 172:588-594.

Ecker V, Knoery C, Rushworth G, et al. 2018. A review of factors associated with maintenance of sinus rhythm after elective electrical cardioversion for atrial fibrillation. Clin Cardiol 41:862-870.

Gillinov AM, Gelijns AC, Parides MK, et al. 2015. Surgical ablation of atrial fibrillation during mitral-valve surgery. N Engl J Med 372:1399-1409.

Grönberg T, Hartikainen JE, Nuotio I, et al. 2015. Can we predict the failure of electrical cardioversion of acute atrial fibrillation? The FinCV study. Pacing Clin Electrophysiol 38:368-375.

Guo Y, Tian Y, Wang H, et al. 2015. Prevalence, incidence, and lifetime risk of atrial fibrillation in China: new insights into the global burden of atrial fibrillation. Chest 147:109-119.

Ishii Y, Gleva MJ, Gamache MC, et al. 2004. Atrial tachyarrhythmias after the maze procedure: incidence and prognosis. Circulation 110:II164-168.

Marchese P, Bursi F, Delle Donne G, et al. 2011. Indexed left atrial volume predicts the recurrence of non-valvular atrial fibrillation after successful cardioversion. Eur J Echocardiogr 12:214-221.

Maroto LC, Carnero M, Silva JA, et al. 2011. Early recurrence is a predictor of late failure in surgical ablation of atrial fibrillation. Interact Cardiovasc Thorac Surg 12:681-686.

Ruel M, Kulik A, Lam BK, et al. 2005. Long-term outcomes of valve replacement with modern prostheses in young adults. Eur J Cardiothorac Surg 27:425-433.

van Breugel HN, Gelsomino S, de Vos CB, et al. 2014. Maintenance of sinus rhythm after electrical cardioversion for recurrent atrial fibrillation following mitral valve surgery with or without associated radiofrequency ablation. Int J Cardiol 175:290-296.

Van Gelder IC, Crijns HJ, Van Gilst WH, Verwer R, Lie KI. 1991. Prediction of uneventful cardioversion and maintenance of sinus rhythm from direct-current electrical cardioversion of chronic atrial fibrillation and flutter. Am J Cardiol 68:41-46.

Voskoboinik A, Moskovitch JT, Harel N, et al. 2017. Revisiting pulmonary vein isolation alone for persistent atrial fibrillation: A systematic review and meta-analysis. Heart Rhythm 14:661-667. 